\title{
Inhibitory effects of FKBP14 on human cervical cancer cells
}

\author{
LIAN-YI SUN, JIU-ZHI TAO, BING YAN and JIAN-SHU LIN \\ Department of Medical Imaging, Jiading Maternal and Child Health Hospital, Shanghai 201821, P.R. China
}

Received June 28, 2016; Accepted April 24, 2017

DOI: $10.3892 / \mathrm{mmr} .2017 .7043$

\begin{abstract}
The FK506-binding protein 14 (FKBP14), which belongs to a subfamily of immunophilins, has been implicated in various biochemical processes. However, its effects on human cervical cancer remain to be elucidated. The present study aimed to determine the exact role of FKBP14 in human cervical cancer cell proliferation, cell cycle progression, apoptosis, invasion and migration. Cell proliferation was measured by Cell Counting Kit- 8 assay. Flow cytometry was conducted to determine the effects of FKBP14 on cell cycle progression and apoptosis. Cell invasion and migration were determined by Transwell assay. The results of the present study demonstrated that silencing FKBP14 expression using short hairpin (sh)RNA suppressed proliferation, invasion and migration of $\mathrm{HeLa}$ and C-33A cells, and also induced apoptosis and cell cycle arrest. Furthermore, silencing FKBP14 expression decreased the protein expression levels of B-cell lymphoma 2 (Bcl-2), matrix metalloproteinase (MMP)2 and MMP9, and increased the levels of caspase- 3 and $\mathrm{Bcl}-2$-associated $\mathrm{X}$ protein in FKBP14 shRNA-infected HeLa and C-33A cells. In conclusion, FKBP14 may act as an oncogene through suppressing apoptosis and promoting motility in human cervical carcinogenesis; therefore, it may be considered a potential therapeutic target for the treatment of cervical cancer.
\end{abstract}

\section{Introduction}

Cervical cancer is one of the leading causes of cancer-associated mortality and remains the second most common female malignant disease worldwide (1). Although the majority of cases of cervical cancer are preventable, it currently affects $\sim 500,000$ women per year and some patients with high risk factors have an unfavorable prognosis (2). The 5-year survival rate of patients with cervical cancer and lymph node metastasis reduced by $20-30 \%$, compared with patients without lymph node metastasis (3). Therefore, identification of the

Correspondence to: Dr Lian-Yi Sun, Department of Medical Imaging, Jiading Maternal and Child Health Hospital, 1216 Gaotai Road, Jiading, Shanghai 201821, P.R. China

E-mail: lianyisunsh@sina.com

Key words: cervical cancer, FKBP14, Bax/Bcl-2, MMP2, MMP9 underlying molecular mechanisms associated with metastasis may contribute to improving the prognosis of cervical cancer.

Cell motility is an essential cellular process associated with cancer cell invasion and metastasis. Cell migration usually occurs in response to a chemoattractant or a growth factor, which is known as chemotaxis (4). In response to a signal, a migrating cell enters the cell motility cycle in an amoeboid-like manner. Previous studies have aimed to improve understanding regarding the molecular mechanisms underlying cell motility, in an attempt to identify novel therapeutic targets that may inhibit tumor invasion and migration $(5,6)$. Gene therapy is a targeted technique that may be used to treat cancer, and various studies have been conducted to investigate the genes that are involved in metastasis of cervical cancer $(7,8)$. However, the highly complex molecular mechanism underlying metastasis remains poorly understood.

FK506-binding protein 14 (FKBP14) belongs to a family of highly conserved proteins known as immunophilins, which bind to the immunosuppressive drug FK506 and often possess peptidyl-prolyl cis-trans isomerase activity $(9,10)$. FKBPs are present in various organisms and have been implicated in numerous biochemical activities, including protein folding, receptor signaling, protein trafficking and transcription $(11,12)$. Although growing evidence has suggested the important roles of various FKBPs, knowledge of their specific functions remains unclear. In particular, to the best of our knowledge, there is little information regarding the roles that FKBPs serve in the development and progression of cancer. According to Oncomine (www.oncomine.org/resource/login. html), FKBP5 is overexpressed in brain cancer, prostate cancer, lymphoma, head and neck cancer, and melanoma (13). Conversely, FKBP5 has been reported to be downregulated in pancreatic cancer, colon cancer and testicular cancer $(13,14)$. Furthermore, FKBP38 has been reported to induce the localization of the anti-apoptotic proteins, B-cell lymphoma 2 (Bcl-2) and Bcl-extralarge, to the mitochondrial membrane and protect cells against apoptosis (15). Previous studies have demonstrated that FKBP14 is overexpressed in ovarian cancer and metastatic prostate cancer $(16,17)$.

The present study aimed to determine the precise role of FKBP14 in human cervical cancer cell proliferation, cell cycle progression, apoptosis, invasion and migration. The present study indicated that FKBP14 is overexpressed in human cervical cancer cell lines. Notably, FKBP14 knockdown markedly inhibited HeLa and C-33A cell proliferation, invasion and migration, and induced cell cycle arrest and apoptosis. Furthermore,FKBP14 is associated with cell apoptosis, invasion 
and migration via the regulation ofcaspase-3, Bcl-2-associated $\mathrm{X}$ protein $(\mathrm{Bax}) / \mathrm{Bcl}-2$, matrix metalloproteinase $(\mathrm{MMP}) 2$ and MMP9 expression. These findings support a potential role for FKBP14 as a tumor promoter, and suggest that it may have efficacy as a target for the treatment of human cervical cancer.

\section{Materials and methods}

Cell culture. The following human cervical cancer cell lines: HeLa, C4-1, C-33A, SiHa and CaSki in addition to normal cervical epithelial cells (CRL2614) were obtained from the Institute of Biochemistry and Cell Biology (Shanghai, China). Cells were cultured in Dulbecco's modified Eagle's medium (DMEM; Thermo Fisher Scientific Inc., Waltham, MA, USA) supplemented with $10 \%$ fetal bovine serum (FBS, Invitrogen; Thermo Fisher Scientific, Inc.), $100 \mathrm{U} / \mathrm{ml}$ penicillin and $100 \mu \mathrm{g} / \mathrm{ml}$ streptomycin, and were incubated in a humidified atmosphere containing $5 \% \mathrm{CO}_{2}$ at $37^{\circ} \mathrm{C}$.

Reverse transcription-quantitative polymerase chain reaction (RT-qPCR). Total RNA was isolated from cervical cancer cells using TRIzol reagent (Invitrogen; Thermo Fisher Scientific, Inc.) and was detected by $1 \%$ agarose gel electrophoresis to determine quantity and quality. As the template for PCR reactions, cDNA was synthesized from $1 \mathrm{mg}$ RNA using AMV reverse transcriptase (Fermentas; Thermo Fisher Scientific, Inc.) according to the manufacturer's protocol. RT-qPCR was conducted using SYBR-Green ${ }^{\circledR}$ 10X SuperMix (Takara Bio, Inc., Otsu, Japan) in a $25 \mu$ l total volume using a Roche Light Cycler ${ }^{\circledR} 480$ II system (Roche Diagnostics, Basel, Switzerland). The PCR cycling conditions were as follows: $95^{\circ} \mathrm{C}$ for $10 \mathrm{~min}$, followed by 40 cycles at $95^{\circ} \mathrm{C}$ for $15 \mathrm{sec}$ and $60^{\circ} \mathrm{C}$ for $45 \mathrm{sec}$, and a final extension step of $95^{\circ} \mathrm{C}$ for $15 \mathrm{sec}$, $60^{\circ} \mathrm{C}$ for $1 \mathrm{~min}, 95^{\circ} \mathrm{C}$ for $15 \mathrm{sec}$ and $60^{\circ} \mathrm{C}$ for $15 \mathrm{sec}$. Primer pairs for human genes were designed using Primer Express software (version 3.0.1; Applied Biosystems; Thermo Fisher Scientific, Inc.) as follows: FKBP14 forward, 5'-TGAAGG ACAGCACCAATAG-3' and reverse, 5'-GCACATTTACCA CCAACTC-3'; and GAPDH forward, 5'-CACCCACTCCTC CACCTTTG-3' and reverse, 5'-CCACCACCCTGTTGCTGT AG-3'. GAPDH was used as the internal control. All PCR reactions were performed in triplicate and the relative expression levels of FKBP14 were calculated normalized to the mRNA expression levels of GAPDH using the $\Delta \Delta \mathrm{Cq}$ method (18).

RNA interference (RNAi) and construction of stable cell lines. A short hairpin (sh)RNA (shRNA, 5'-GACCACUUU CACUGAUUAU-3'; Sangon Biotech Co., Ltd., Shanghai, China) targeting human FKBP14 mRNA was cloned into the pLVX-AcGFP-C1 lentiviral vector (Sangon Biotech Co., Ltd.) using EcoRI and BamHI. Scramble shRNA (5'-CUGGAUGAA GCUUGACAUUA-3'; Sangon Biotech Co., Ltd.) was cloned into the pLVX-AcGFP-C1 lentiviral vector, and was used as a negative control (shNC). The constructs were subsequently transfected into human embryonic kidney-293T cells (Shanghai Institute of Biochemistry and Cell Biology, Shanghai, China) with pLVX-AcGFP-C1 lentiviral packaging vectors using Lipofectamine 2000 (Invitrogen; Thermo Fisher Scientific, Inc.) according to the manufacturer's protocol. Transfected cells were cultured in DMEM supplemented with $10 \%$ FBS,
$100 \mathrm{U} / \mathrm{ml}$ penicillin and $100 \mu \mathrm{g} / \mathrm{ml}$ streptomycin, and were incubated in a humidified atmosphere containing $5 \% \mathrm{CO}_{2}$ at $37^{\circ} \mathrm{C}$. Viruses were collected by centrifugation at $1,000 \mathrm{x}$ g for $5 \mathrm{~min}$ at $37^{\circ} \mathrm{C} 48 \mathrm{~h}$ post-transfection, and were used to infect HeLa and C-33A cells at a multiplicity of infection of 20 in the presence of $8 \mathrm{mg} / \mathrm{ml}$ polybrene (Sigma-Aldrich; Merck KGaA, Darmstadt, Germany). Subsequent experiments were performed $48 \mathrm{~h}$ post-infection.

Cell proliferation assay. The effects of FKBP14 shRNA on the proliferation of HeLa and C-33A cells were determined by Cell Counting Kit-8 (CCK-8; Dojindo Molecular Technologies, Inc., Rockville, MD, USA) assay. Briefly, $5 \times 10^{4}$ cells were seeded into 96-well culture plates at $100 \mu \mathrm{l} /$ well. Subsequently, cell proliferation was evaluated using the CCK-8 assay according to the manufacturer's protocol. The absorbance of each supernatant was measured at a wavelength of $450 \mathrm{~nm}$ using a microplate reader (Thermo Fisher Scientific, Inc.).

Flow cytometry. For cell cycle analysis, FKBP14 shRNA-infected HeLa and C-33A cells were seeded into 12 -well plates at a density of $3 \times 10^{3}$ cells/well, and the percentage of cells in the various phases of the cell cycle were evaluated by determining the DNA content after propidium iodide (PI) staining. Briefly, cells were washed with PBS, trypsinized and centrifuged at $400 \mathrm{x} \mathrm{g}$ for $5 \mathrm{~min}$ at $4^{\circ} \mathrm{C}$. Pellets were fixed overnight in $70 \%$ cold ethanol. Following fixation, cells were washed twice with PBS and incubated in PBS containing RNase $(1 \mathrm{mg} / \mathrm{ml})$ for $10 \mathrm{~min}$ at room temperature. Finally, samples were stained with PI $(1 \mathrm{mg} / \mathrm{ml})$ for $30 \mathrm{~min}$ at $4^{\circ} \mathrm{C}$. Data acquisition was performed by flow cytometry (BD Accuri C6; software version 1.0.264.21; BD Biosciences, Franklin Lakes, NJ, USA). To analyze apoptosis, an Annexin V-fluorescein isothiocyanate (FITC)/PI double stain assay (BioVision, Inc., Milpitas, CA, USA) was conducted according to the manufacturer's protocol. Floating and trypsinized adherent cells ( $3 \times 10^{3}$ cells/well; 12-well plates) were collected, resuspended in $200 \mu \mathrm{l}$ binding buffer containing $195 \mu \mathrm{l}$ Annexin V-FITC and $5 \mu \mathrm{PI}$, and were incubated for $10 \mathrm{~min}$ in the dark at room temperature prior to flow cytometric analysis (BD Accuri C6, software version 1.0.264.21; BD Biosciences).

Transwell assay. A total of $5 \times 10^{5}$ cells infected with FKBP14 shRNA were plated into the upper wells of a Transwell chamber (Greiner Bio-One GmbH, Frickenhausen, Germany), which was precoated with Matrigel (BD Biosciences) for the invasion assay and was uncoated for the migration assay. In both assays, the lower wells were filled with $600 \mu$ l DMEM containing $10 \% \mathrm{FBS}$. After $48 \mathrm{~h}$ at $37^{\circ} \mathrm{C}$, cells in the upper wells were removed using a cotton bud. The cells attached to the lower surface were washed with PBS, fixed in $4 \%$ paraformaldehyde for $10 \mathrm{~min}$ at room temperature and stained with $0.5 \%$ crystal violet for $30 \mathrm{~min}$ at room temperature. Images of the cells were captured and cell numbers were counted under a light microscope (magnification, x200; Olympus Corporation, Tokyo, Japan).

Western blot analysis. Human cervical cancer cell lines were harvested and washed twice with PBS, after which the cells were lysed in ice-cold radioimmunoprecipitation assay buffer 
A

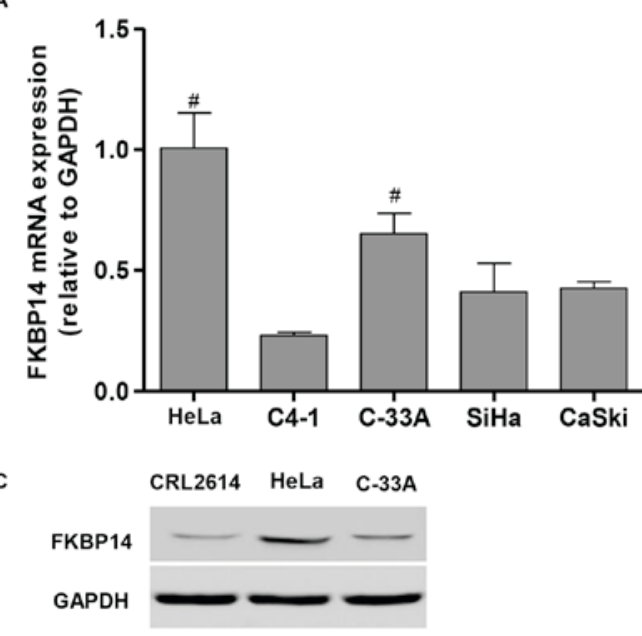

D

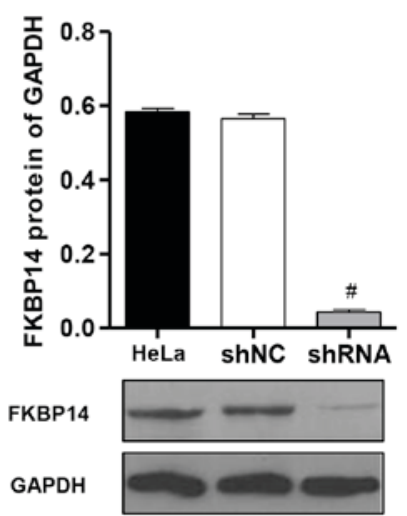

B
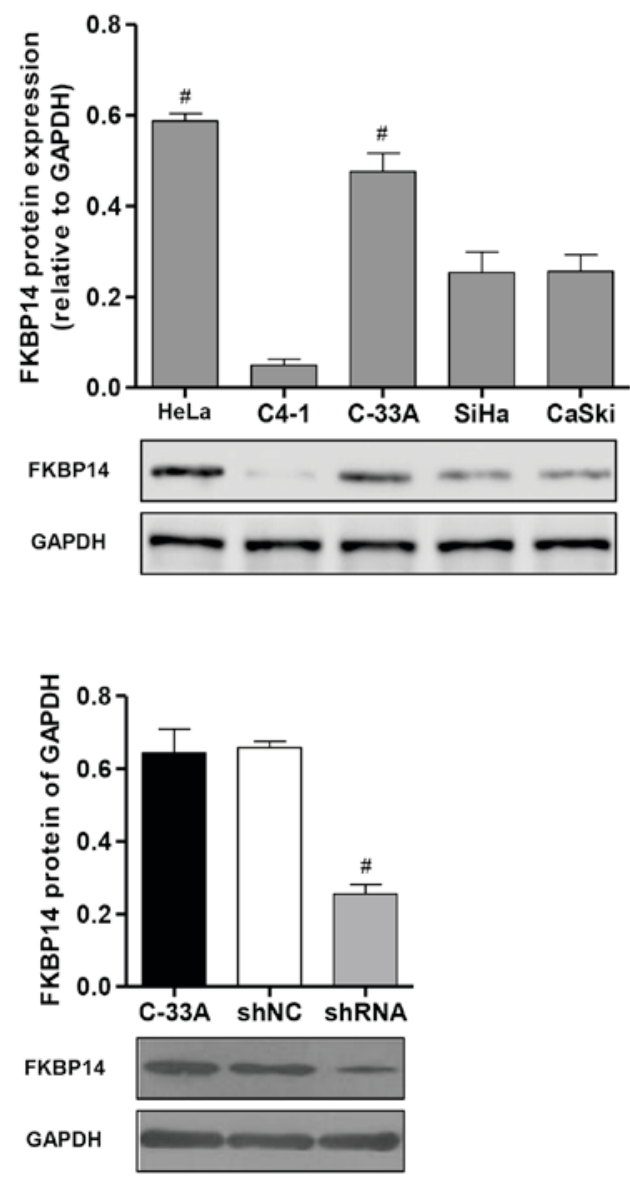

Figure 1. Upregulation of FKBP14 expression in human cervical cancer cell lines. (A) mRNA expression levels of FKBP14 were detected by reverse transcription-quantitative polymerase chain reaction. FKBP14 mRNA expression was significantly increased in HeLa and C-33A cells compared within the three other cervical cancer cell lines. ${ }^{.} \mathrm{P}<0.001$ vs. the four other cervical cancer cell lines. (B) Protein expression levels of FKBP14 were higher in HeLa and C-33A cells compared within the three other cervical cancer cell lines, as detected by western blotting. (C) Protein expression levels of FKBP14 were increased in HeLa and C-33A cells compared with normal cervical epithelial cells (CRL2614), as detected by western blotting. A total of $48 \mathrm{~h}$ post-infection of HeLa and C-33A cells with FKBP14 shRNA, the protein expression levels of FKBP14 were significantly decreased compared within the control (D) HeLa and (E) C-33A cells, as detected by western blotting. ${ }^{~} \mathrm{P}<0.001$ vs. the corresponding control. FKBP14, FK506-binding protein 14; shNC, negative control shRNA; shRNA, short hairpin RNA.

(Beyotime Institute of Biotechnology, Shanghai, China) and were incubated on ice for $30 \mathrm{~min}$. The protein concentration was measured using a bicinchoninic acid protein assay kit (Pierce; Thermo Fisher Scientific, Inc.), and absorbance was measured using a microplate reader (SM600 Labsystem; Shanghai Utrao Medical Instrument Co., Ltd., Shanghai, China). The cell lysates were centrifuged at $13,000 \mathrm{x} \mathrm{g}$ for $10 \mathrm{~min}$ at $4^{\circ} \mathrm{C}$ and the supernatant $(20-30 \mu \mathrm{g}$ protein) was separated by $10 \%$ SDS-PAGE. Proteins were then electrophoretically transferred onto a polyvinylidene fluoride membrane (EMD Millipore, Shanghai, China). The blots were blocked with $5 \%$ skim milk, followed by incubation with specific antibodies against FBKP14 (cat no. ab105018; 1:1,000), cleaved caspase-3 (cat no. ab32351; 1:5,000), Bax (cat no. ab53154; 1:1,000), Bcl-2 (cat no. ab59348; 1:1,000), MMP2 (cat no. ab37150; 1:500) and MMP9 (cat no. ab38898; 1:1,000) (all from Abcam, Cambridge, MA, USA), and GAPDH (cat no. 5174; 1:1,500; Cell Signaling Technology, Inc., Danvers, MA, USA), followed by incubation with horseradish peroxidase-conjugated goat anti-rabbit/anti-mouse secondary antibodies (cat nos. A0208 and A0216; dilution 1:1,000;
Beyotime Institute of Biotechnology) for $1 \mathrm{~h}$ at $37^{\circ} \mathrm{C}$, prior to being washed three times with Tris-buffered saline containing 20\% Tween-20 (Amresco, Solon, OH, USA). The blots were visualized using enhanced chemiluminescence (EMD Millipore, Billerica, MA, USA) and signals were quantified by densitometry (Quantity One software; version 4.62; Bio-Rad Laboratories, Inc., Hercules, CA, USA).

Statistical analysis. All values are presented as the mean \pm standard deviation. All experiments were performed in triplicate. Data were analyzed by unpaired, two-tailed Student's t-test and one-way analysis of variance with Tukey's post hoc test, using Graphpad Prism 6.0 software (GraphPad, Inc., La Jolla, CA, USA). P $<0.05$ was considered to indicate a statistically significant difference.

\section{Results}

FKBP14 expression in human cervical cancer cell lines. The present study initially examined the expression levels of FKBP14 in five human cervical cancer cell lines: HeLa, 
A

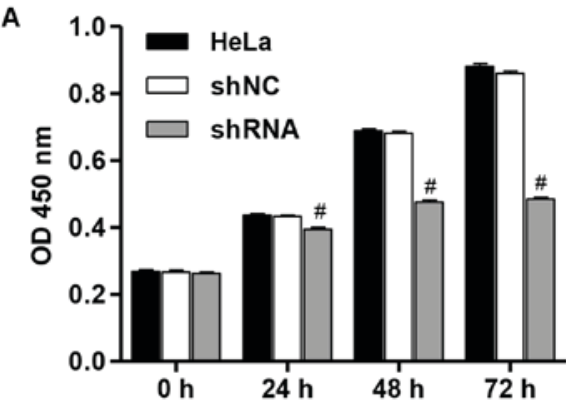

C
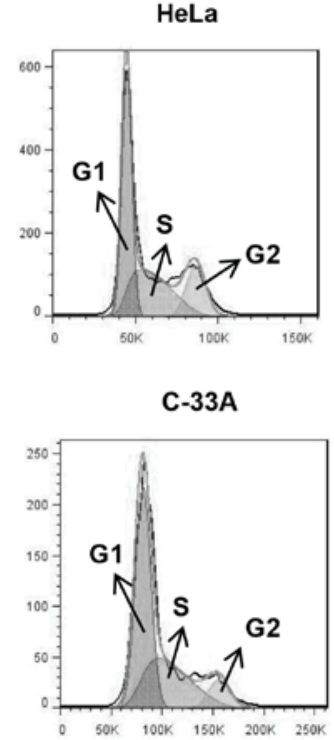

E

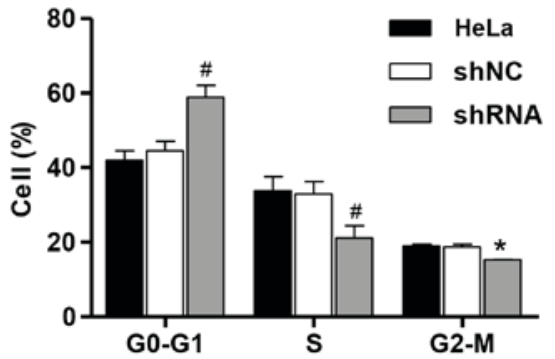

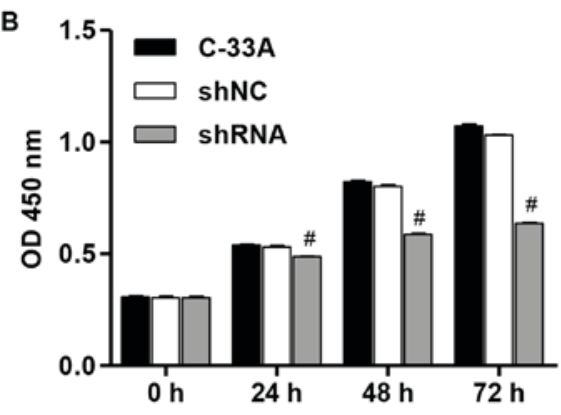

$\operatorname{shNC}$
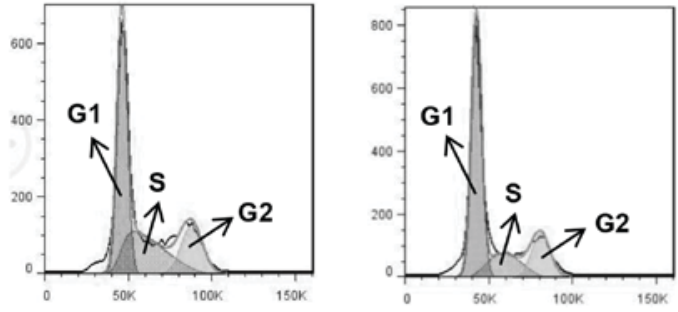

shNC

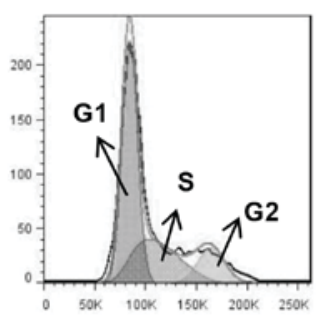

$\mathbf{F}$

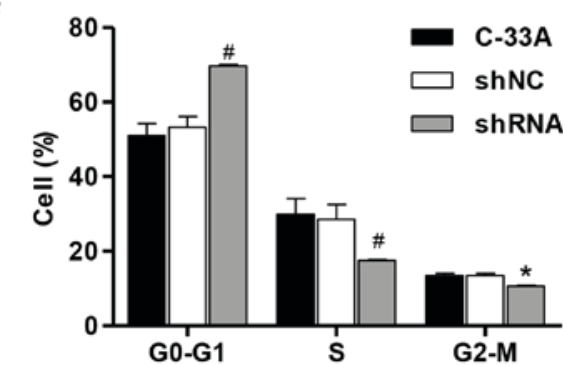

Figure 2. Proliferation and cell cycle progression of HeLa and C-33A cells post-infection with FKBP14 shRNA. A total of 48 h post-infection with FKBP14 shRNA, proliferation was measured by Cell Counting kit-8 assay in (A) HeLa and (B) C-33A cells. (C-F) Cell cycle progression was measured by flow cytometry in $(\mathrm{C}$ and $\mathrm{E}) \mathrm{HeLa}$ and $(\mathrm{D}$ and $\mathrm{F}) \mathrm{C}-33 \mathrm{~A}$ cells. ${ }^{*} \mathrm{P}<0.001,{ }^{*} \mathrm{P}<0.01$ vs. the corresponding control. FKBP14, FK506-binding protein 14 ; shNC, negative control shRNA; shRNA, short hairpin RNA; OD, optical density.

C4-1, C-33A, SiHa and CaSki, by RT-qPCR and western blot analysis, using GAPDH as a reference. The protein expression of FKBP14 in normal cervical epithelial cells (CRL2614) was additionally detected by western blot analysis. The results demonstrated that the mRNA and protein expression levels of FKBP14 were significantly higher in HeLa and C-33A cells compared with the three other cervical cancer cell lines (Fig. 1A and B). Notably, the protein expression of FKBP14 was significantly increased in HeLa and C-33A cells compared with CRL2614 cells (Fig. 1C). Therefore, the FKBP14-specificshRNA was used to infect HeLa and C-33A cells. The shNC-infected HeLa and C-33A cells were used as negative controls, and uninfected HeLa and C-33A cells were used as controls. As presented in Fig. 1D and E, the protein expression levels of FKBP14 were successfully reduced to $\sim 92.6$ and $60.3 \%$ of the levels in the control HeLa and C-33A cells $48 \mathrm{~h}$ post-infection, respectively. In addition, the expression of FKBP14 in the shNC-infected cells was the same as in control HeLa and C-33A cells.

FKBP14 knockdown inhibits cell proliferation and induces cell cycle arrest. The CCK- 8 assay revealed that FKBP14 knockdown significantly inhibited cell proliferation in a time-dependent manner in FKBP14 shRNA-infected HeLa and C-33A cells. Cell proliferation was decreased by $9.8,30.9$ and $45.0 \%$ at 24,48 and $72 \mathrm{~h}$, respectively, compared with the control HeLa group (Fig. 2A). Cell proliferation was decreased by $9.5,28.6$ and $40.7 \%$ at 24,48 and $72 \mathrm{~h}$, respectively, compared with the control C-33A group (Fig. 2B).

Subsequently, the potential inhibitory effects of FKBP14 knockdown on cell cycle progression were investigated. As presented in Fig. 2C-F, suppression of FKBP14 resulted in 

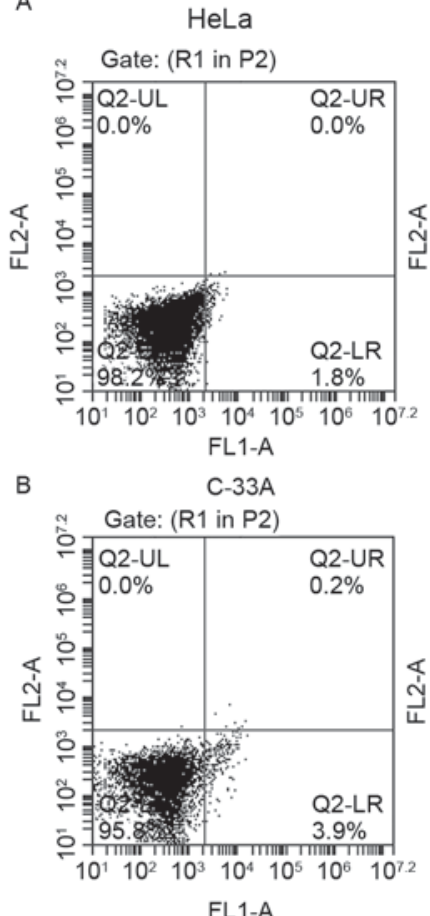
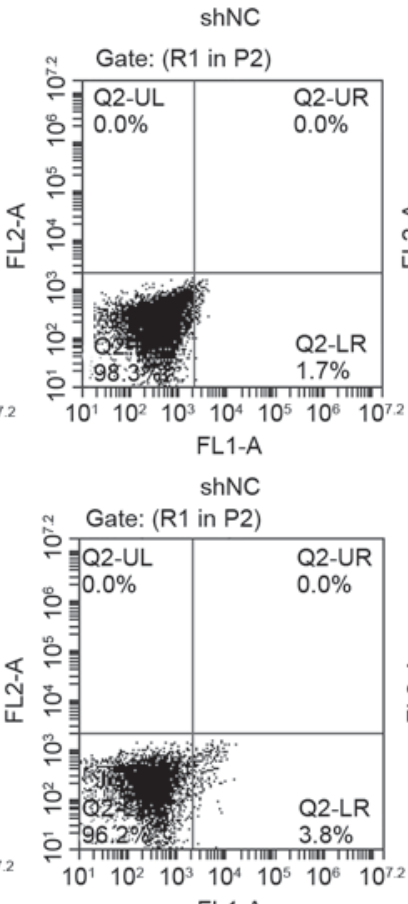
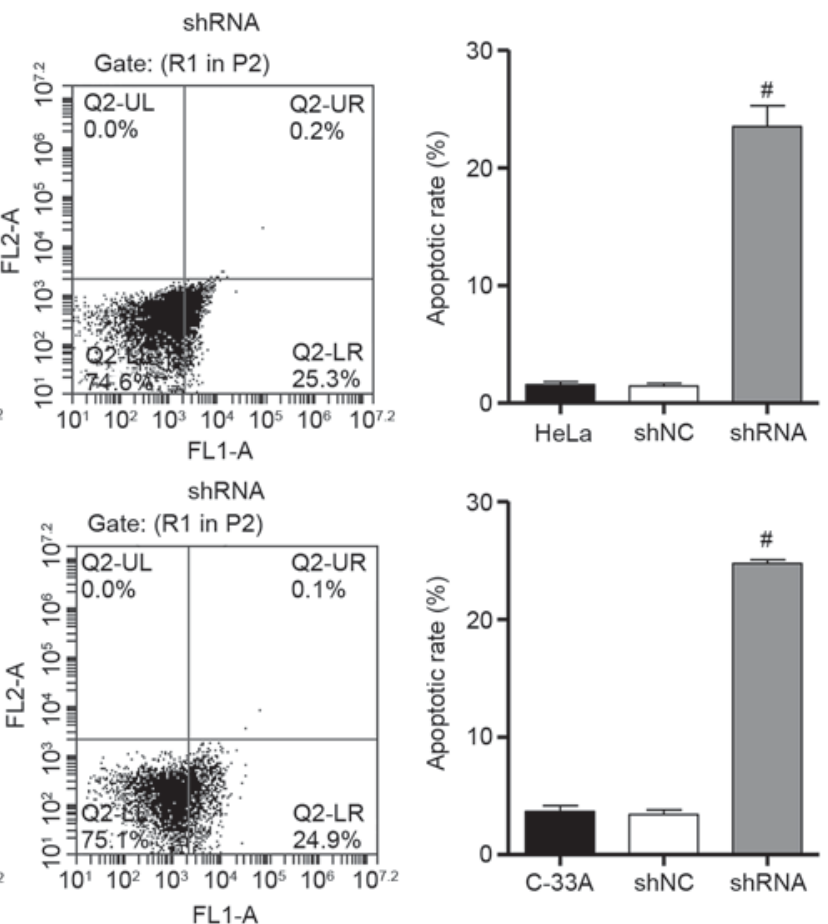

Figure 3. Apoptosis of HeLa and C-33A cells post-infection with FKBP14 shRNA. A total of 48 h post-infection with FKBP14 shRNA, cell apoptosis was measured by flow cytometry in (A) HeLa and (B) C-33A cells. "P<0.001 vs. the corresponding control. FKBP14, FK506-binding protein 14; shNC, negative control shRNA; shRNA, short hairpin RNA.

an increased number of cells in $\mathrm{G}_{1}$ phase (HeLa, mean=58.9; C-33A, mean=69.7) compared with the corresponding controls (HeLa, mean=41.9; C-33A, mean=51.05; $\mathrm{P}<0.001)$. There was a concomitant reduction in the number of cells in $\mathrm{S}$ and $\mathrm{G}_{2} / \mathrm{M}$ phases in HeLa and C-33A cells infected with FKBP14 shRNA. These data suggested that knockdown of FKBP14 induces cell cycle arrest at $G_{1}$ phase in human cervical cancer cell lines, which may be associated with the inhibition of cell proliferation.

FKBP14 knockdown induces cell apoptosis. To assess the effects of FKBP14 on cell apoptosis, Annexin V/PI staining was performed. The mean apoptotic rate was significantly increased to 23.5 and $24.7 \%$ in FKBP14 shRNA-infected HeLa and C-33A cells, respectively, compared with in the control groups (HeLa, mean $=1.6 \%$; C-33A, mean $=3.7 \%$, $\mathrm{P}<0.001$; Fig. 3). These results suggested that FKBP14 may serve an anti-apoptotic role in human cervical cancer cell lines, as indicated by FKBP14 knockdown-induced inhibition of cell proliferation and induction of cell cycle arrest.

FKBP14 knockdown inhibits cell invasion and migration. The effects of FKBP14 knockdown on cervical cancer cell invasion and migration were determined by Transwell assay. As presented in Fig. 4, FKBP14 shRNA markedly suppressed the invasion of HeLa and C-33A cells by 46.1 and $56.1 \%$, respectively, compared with the HeLa and C-33A control groups. The ability of cells to migrate across uncoated chambers was also measured. The number of migratory FKBP14 shRNA-infected HeLa and C-33A cells was significantly decreased by 41.7 and $50.1 \%$ compared with the HeLa and C-33A control groups (Fig. 5).
FKBP14 knockdown regulates the expression of apoptosis-, invasion-and migration-associated proteins. The present study examined the expression levels of proteins associated with cell apoptosis, invasion and migration by western blot analysis. The expression levels of apoptosis-associated proteins, including caspase- 3 and Bax, were significantly increased by 22.2 and $43.4 \%$, respectively, whereas Bcl-2 was significantly decreased by $92.7 \%$, in FKBP14 shRNA-infected HeLa cells compared with the control HeLa cells (Fig. 6A). In addition, the expression levels of proteins involved in cell invasion and migration, MMP2 and MMP9, were significantly decreased by 73.2 and $86.9 \%$, respectively, in FKBP14 shRNA-infected HeLa cells compared with the control HeLa cells (Fig. 6A). In FKBP14 shRNA-infected C-33A cells, the protein expression levels of caspase-3 and Bax were increased by 68.3 and $85.1 \%$, whereas the protein expression levels of Bcl-2, MMP2 and MMP9 were decreased by 40.5, 43.2 and $57.1 \%$, respectively, in FKBP14 shRNA-infected cells compared with the control C-33A cells (Fig. 6B). These findings suggested that the effects of FKBP14 on cervical cancer cell apoptosis, invasion and migration may be associated with regulation of the protein expression levels of caspase-3, Bax, Bcl-2, MMP2 and MMP9.

\section{Discussion}

Cervical cancer is the most prevalent malignancy of the female reproductive system. Although great progress has been achieved, current therapies for the treatment of patients with advanced cervical cancer are still unfavorable, due to uncontrolled local cancer, recurrence and/or distant metastasis (19). Members of the FKBP family have been implicated in various processes, particularly in cancer, including cell 


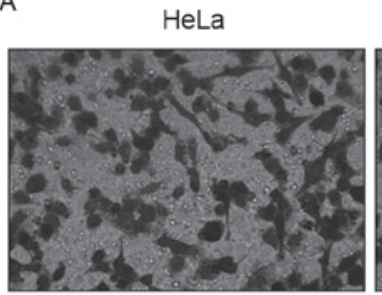

B

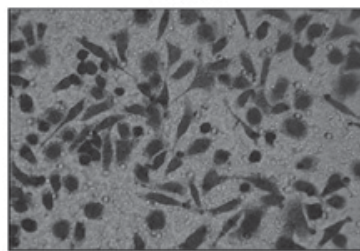

shNC

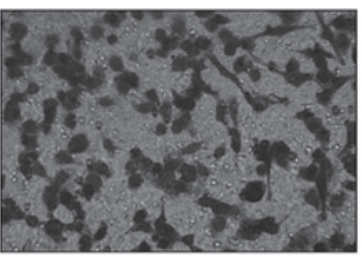

shNC

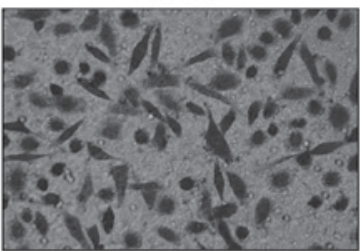

shRNA

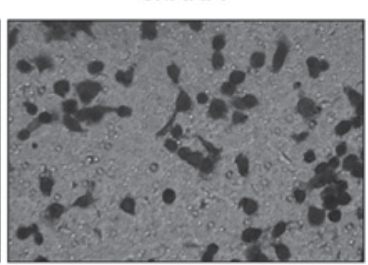

shRNA

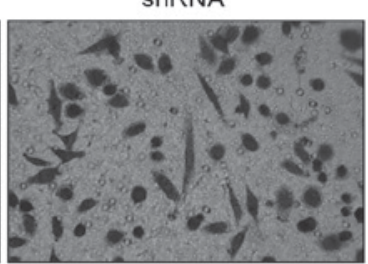

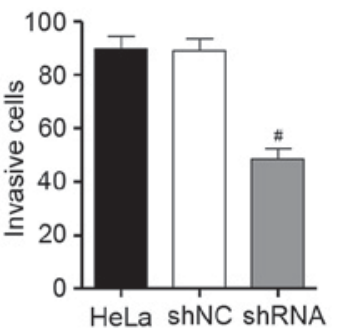

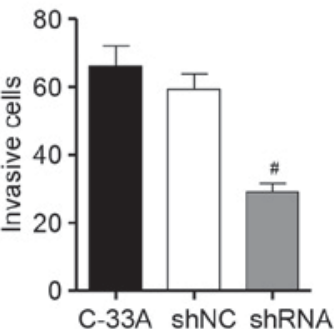

Figure 4. Invasive abilities of HeLa and C-33A cells post-infection with FKBP14 shRNA. A total of 48 h post-infection with FKBP14 shRNA, cell invasion was measured by Transwell assay in (A) HeLa and (B) C-33A cells. ${ }^{\#} \mathrm{P}<0.001$ vs. the corresponding control. FKBP14, FK506-binding protein 14; shNC, negative control shRNA; shRNA, short hairpin RNA.

A

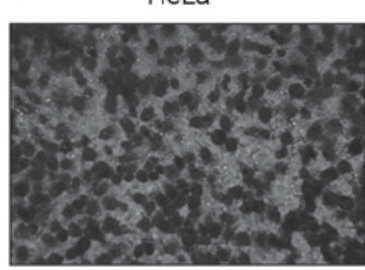

B

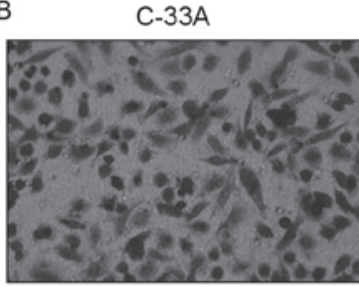

$\operatorname{shNC}$

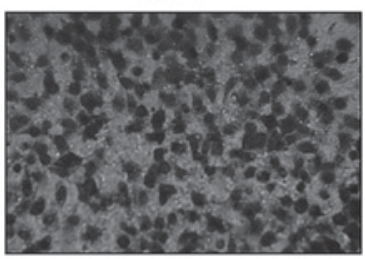

$\operatorname{shNC}$

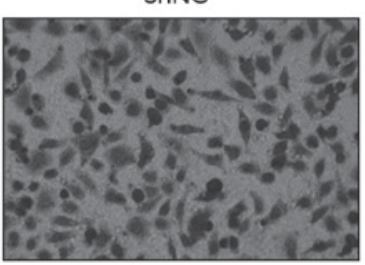

ShRNA

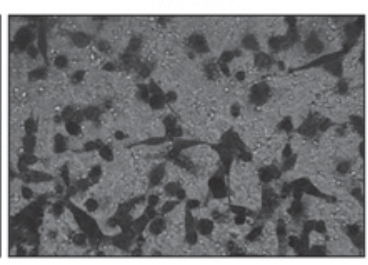

ShRNA

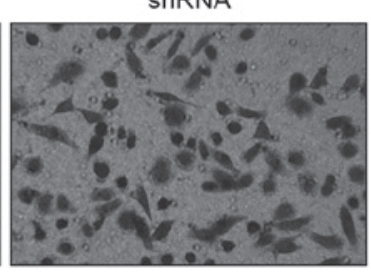

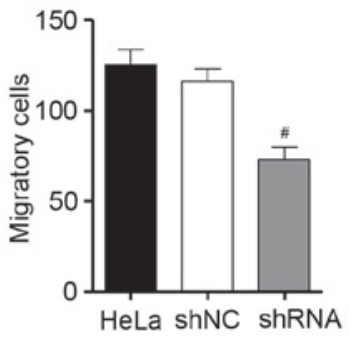

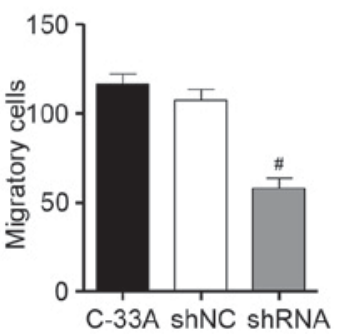

Figure 5. Migratory abilities of HeLa and C-33A cells post-infection with FKBP14 shRNA. A total of 48 h post-infection with FKBP14 shRNA, cell migration was measured by Transwell assayin (A) HeLa and (B) C-33A cells. ${ }^{*} \mathrm{P}<0.001$ vs. the corresponding control. FKBP14, FK506-binding protein 14; shNC, negative control shRNA; shRNA, short hairpin RNA.

cycle progression, and survival and apoptotic signaling pathways (20). Since dysregulation of FKBP expression has been observed in cancer tissues, it has been hypothesized that FKBP members serve an important role in tumorigenesis and the response to chemotherapy and radiotherapy; in addition, it has been suggested that FKBP members may act as oncogenes or tumor suppressors depending on the tissue type. A previous study reported that FKBP14 is frequently upregulated in various human malignancies (21). In the present study, the mRNA and protein expression levels of FKBP14 were higher in HeLa and C-33A cells compared with in three other human cervical cancer cell lines.

To investigate the roles of FKBP14 in cervical cancer cells, RNAi was conducted. The results of the present study demonstrated that FKBP14 knockdown significantly inhibited cell proliferation, and induced cell cycle arrest and apoptosis, in HeLa and C-33A cells. Other members of the FKBP family have also been reported to be involved in the regulation of cell cycle progression and apoptosis. For example, cells from FKBP12-deficient mice exhibited cell cycle arrest in $\mathrm{G}_{1}$ phase (22). Furthermore, overexpression of FKBP38 resulted in the suppression of apoptosis, whereas functional inhibition of this protein promoted cell apoptosis (23). Cleaved caspase-3 is a well-known marker for cell apoptosis (24). The Bcl-2 family proteins exert anti-apoptotic (e.g. Bcl-2) or proapoptotic (e.g. Bax) effects, and the ratio of $\mathrm{Bax} / \mathrm{Bcl}-2$ is a useful index to evaluate cell apoptosis (25). The present study revealed that knockdown of FKBP14 increased the expression of cleaved caspase-3 and the ratio of $\mathrm{Bax} / \mathrm{Bcl}-2$, thus suggesting that FKBP14 may exert an inhibitory effect on cell apoptosis by regulating caspase- 3 expression and the ratio of $\mathrm{Bax} / \mathrm{Bcl}-2$.

Invasion and metastasis are important malignancy-associated biological behaviors, which are the main causes of mortality in patients with advanced cervical cancer (26). 
A
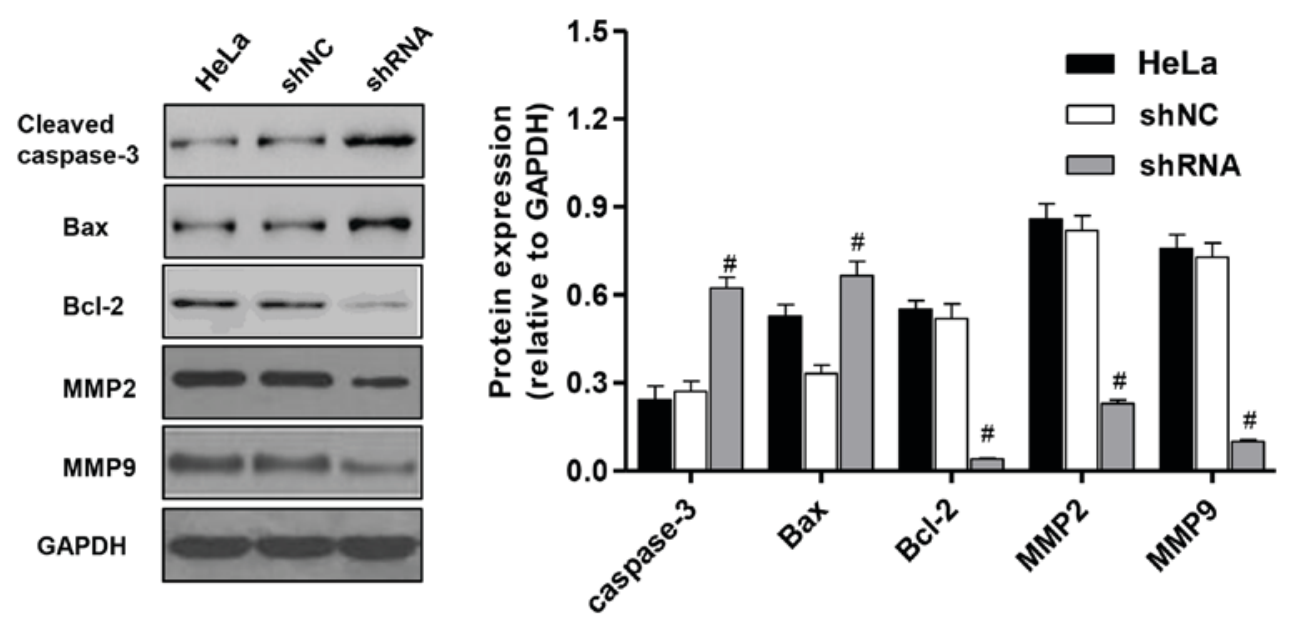

B
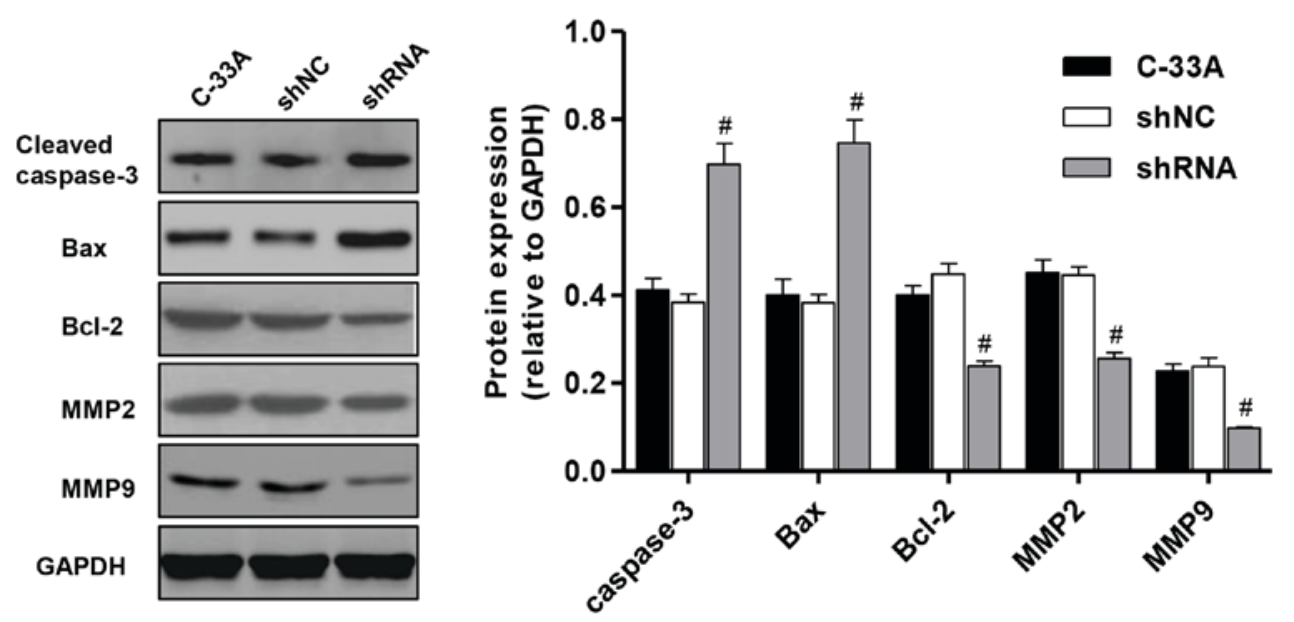

Figure 6. Protein expression in HeLa and C-33A cells post-infection with FKBP14 shRNA. A total of 48 h post-infection with FKBP14 shRNA, the protein expression levels of cleaved caspase-3, Bax, Bcl-2, MMP2 and MMP9 were measured by western blotting in (A) HeLa and (B) C-33A cells. ${ }^{*} \mathrm{P}<0.001$ vs. the corresponding control. FKBP14, FK506-binding protein 14; shNC, negative control shRNA; shRNA, short hairpin RNA; Bcl-2, B-cell lymphoma 2; Bax, Bcl-2-associated X protein; MMP, matrix metalloproteinase.

Active cell migration is a critical factor in the invasion and metastasis of cancer, and highly invasive cancer cells usually exhibit more active cell migration. In the present study, the effects of FKBP14 shRNA infection on the abilities of HeLa and C-33A cells to invade and migrate were determined by Transwell assay. The results indicated that the number of invasive and migratory HeLa and C-33A cells was significantly decreased following FKBP14 knockdown. Degradation and destruction of the extracellular matrix and basement membrane are crucial steps in the invasion and metastasis of cancer, in which MMPs serve an important role $(27,28)$. Two of these enzymes, MMP2 and MMP9, are associated with tumor invasion and metastasis. Overexpression of MMP2 and MMP9 has been detected in pre-cancer and cancerous lesions of the cervix (29). Previous studies have reported that MMP9 is involved in cancer angiogenesis via vascular endothelial growth factor regulation, and serves a central role in the cleavage of certain cytokine receptors on tumor-infiltrating lymphocytes derived from human cervical cancer $(30,31)$. The results of the present study indicated that the expression levels of MMP2 and MMP9 were decreased in FKBP14 shRNA-infected HeLa and C-33A cells. These findings indicated that FKBP14 promotes invasion and migration by modulating the expression of MMP2 and MMP9.

In conclusion, further studies regarding the association of FKBP14 with apoptosis, invasion and migration of human cervical cancer are required to determine the exact mechanism underlying the oncogenic effects of FKBP14.

\section{References}

1. Barra F, Lorusso D, Leone Roberti Maggiore U, Ditto A, Bogani G, Raspagliesi $\mathrm{F}$ and Ferrero S: Investigational drugs for the treatment of cervical cancer. Expert Opin Investig Drugs 26: 389-402, 2017.

2. Biewenga P, van der Velden J, Mol BW, Stalpers LJ, Schilthuis MS, van der Steeg JW, Burger MP and Buist MR: Prognostic model for survival in patients with early stage cervical cancer. Cancer 117: 768-776, 2011.

3. Wang F, Li Y, Zhou J, Xu J, Peng C, Ye F, Shen Y, Lu W, Wan X and Xie X: miR-375 is down-regulated in squamous cervical cancer and inhibits cell migration and invasion via targeting transcription factor SP1. Am J Pathol 179: 2580-2588, 2011.

4. Hanna S and El-Sibai M: Signaling networks of Rho GTPases in cell motility. Cell Signal 25: 1955-1961, 2013.

5. Sharma C, Nusri Qel-A, Begum S, Javed E, Rizvi TA and Hussain A: (-)-Epigallocatechin-3-gallate induces apoptosis and inhibits invasion and migration of human cervical cancer cells. Asian Pac J Cancer Prev 13: 4815-4822, 2012. 
6. Ramachandran I, Thavathiru E, Ramalingam S, Natarajan G Mills WK, Benbrook DM, Zuna R, Lightfoot S, Reis A, Anant S and Queimado L: Wnt inhibitory factor 1 induces apoptosis and inhibits cervical cancer growth, invasion and angiogenesis in vivo. Oncogene 31: 2725-2737, 2012.

7. Kodama J,Hasengaowa, Kusumoto T, Seki N, Matsuo T, Ojima Y, Nakamura K, Hongo A and Hiramatsu Y: Association of CXCR4 and CCR7 chemokine receptor expression and lymph node metastasis in human cervical cancer. Ann Oncol 18: 70-76, 2007.

8. Kim HJ, Do IG, Jeon HK, Cho YJ, Park YA, Choi JJ, Sung CO, Lee YY, Choi CH, Kim TJ, et al: Galectin 1 expression is associated with tumor invasion and metastasis in stage IB to IIA cervical cancer. Hum pathol 44: 62-68, 2013.

9. Barik S: Immunophilins: For the love of proteins. Cell Mol Life Sci 63: 2889-2900, 2006.

10. Kang CB, Hong Y, Dhe-Paganon S and Yoon HS: FKBP family proteins: Immunophilins with versatile biological functions Neurosignals 16: 318-325, 2008.

11. McKeen HD, McAlpine K, Valentine A, Quinn DJ, McClelland K, Byrne C, O'Rourke M, Young S, Scott CJ, McCarthy HO, et al: A novel FK506-like binding protein interacts with the glucocorticoid receptor and regulates steroid receptor signaling. Endocrinology 149: 5724-5734, 2008.

12. Nigam N, Singh A, Sahi C, Chandramouli A and Grover A: SUMO-conjugating enzyme (Sce) and FK506-binding protein (FKBP) encoding rice (Oryza sativa L.) genes: Genome-wide analysis, expression studies and evidence for their involvement in abiotic stress response. Mol Genet Genomics 279: 371-383, 2008.

13. Li L, Lou Z and Wang L: The role of FKBP5 in cancer aetiology and chemoresistance. Br J Cancer 104: 19-23, 2011.

14. Pei H, Li L, Fridley BL, Jenkins GD, Kalari KR, Lingle W, Petersen G, Lou Z and Wang L: FKBP51 affects cancer cell response to chemotherapy by negatively regulating Akt. Cancer cell 16: 259-266, 2009.

15. Kang CB, Feng L, Chia J and Yoon HS: Molecular characterization of FK-506 binding protein 38 and its potential regulatory role on the anti-apoptotic protein Bcl-2. Biochem Biophys Res Commun 337: 30-38, 2005.

16. Coveney C, Boocock DJ, Rees RC, Deen S and Ball GR: Data mining of gene arrays for biomarkers of survival in ovarian cancer. Microarrays (Basel) 4: 324-338, 2015.

17. Abou-Ouf H, Alshalalfa M, Bristow RG, Argiropoulos B and Bismar TA: Identification and validation of potential driver genes in human prostate cancer: An integrative oncogenomic approach, 2015.
18. Livak KJ and Schmittgen TD: Analysis of relative gene expression data using real-time quantitative PCR and the 2(-Delta Delta C(T)) method. Methods 25: 402-408, 2001

19. Sun Y, Liu JH, Sui YX, Jin L, Yang Y, Lin SM and Shi H: Beclin1 overexpression inhibitis proliferation, invasion and migration of CaSki cervical cancer cells. Asian Pac J Cancer Prev 12: 1269-1273, 2011.

20. Solassol J, Mange A and Maudelonde T: FKBP family proteins as promising new biomarkers for cancer. Curr Opin Pharmacol 11: 320-325, 2011.

21. Halatsch ME, Löw S, Hielscher T, Schmidt U, Unterberg A and Vougioukas VI: Epidermal growth factor receptor pathway gene expressions and biological response of glioblastoma multiforme cell lines to erlotinib. Anticancer res 28: 3725-3728, 2008.

22. Aghdasi B, Ye K, Resnick A, Huang A, Ha HC, Guo X, Dawson TM, Dawson VL and Snyder SH: FKBP12, the 12-kDa FK506-binding protein, is a physiologic regulator of the cell cycle. Proc Natl Acad Sci USA 98: 2425-2430, 2001.

23. Shirane $M$ and Nakayama KI: Inherent calcineurin inhibitor FKBP38 targets $\mathrm{Bcl}-2$ to mitochondria and inhibits apoptosis. Nat Cell Biol 5: 28-37, 2003.

24. Porter AG and Jänicke RU: Emerging roles of caspase-3 in apoptosis. Cell Death Differ 6: 99-104, 1999.

25. Ola MS, Nawaz M and Ahsan H: Role of Bcl-2 family proteins and caspases in the regulation of apoptosis. Mol Cell Biochem 351: $41-58,2011$.

26. Valastyan S and Weinberg RA: Tumor metastasis: Molecular insights and evolving paradigms. Cell 147: 275-292, 2011.

27. Deryugina EI and Quigley JP: Matrix metalloproteinases and tumor metastasis. Cancer Metastasis Rev 25: 9-34, 2006.

28. Gialeli C, Theocharis AD and Karamanos NK: Roles of matrix metalloproteinases in cancer progression and their pharmacological targeting. FEBS J 278: 16-27, 2011.

29. Libra M, Scalisi A, Vella N, Clementi S, Sorio R, Stivala F, Spandidos DA and Mazzarino C: Uterine cervical carcinoma: Role of matrix metalloproteinases (Review). Int J Oncol 34: 897-903, 2009.

30. Kim SH, Cho NH, Kim K, Lee JS, Koo BS, Kim JH, Chang JH and Choi EC: Correlations of oral tongue cancer invasion with matrix metalloproteinases (MMPs) and vascular endothelial growth factor (VEGF) expression. J Surg Oncol 93: 330-337, 2006.

31. Sheu BC, Hsu SM, Ho HN, Lien HC, Huang SC and Lin RH: A novel role of metalloproteinase in cancer-mediated immunosuppression. Cancer res 61: 237-242, 2001. 